\title{
Randomizing Endowments: An Experimental Study of Rational Expectations and Reference-Dependent Preferences ${ }^{\top}$
}

\author{
By Annette Cerulli-Harms, Lorenz Goette, and Charles Sprenger*
}

\begin{abstract}
We test expectations-based reference dependence in market experiments with probabilistic forced exchange. Köszegi and Rabin (2006) predict that when the probability of forced exchange increases, individuals cannot expect to stick with the status quo, and should grow more willing to exchange. This mechanism may eliminate and even reverse the "endowment effect" (Knetsch and Sinden 1984; Kahneman, Knetsch, and Thaler 1990). In a series of experiments with overall 930 subjects, we show some tentative support for the notion that attitudes toward exchange are influenced by the probability of forced exchange. However, the results are sensitive to small changes in experimental design. (JEL C92, D12, D84, D91)
\end{abstract}

\begin{abstract}
Tószegi and Rabin (2006, 2007, 2009-henceforth, KR) provide an elegant way _ of disciplining models of reference dependence by assuming that reference points are given by expectations. 1 The KR model is able to accommodate many of the observed anomalies attributed to standard reference dependence, and has also spurred new applications in macroeconomics (Pagel 2016, 2017), industrial organization (Heidhues and Köszegi 2008, 2014), and contract theory (de Meza and Webb 2007; Herweg, Müller, and Weinschenk 2010).
\end{abstract}

\footnotetext{
* Cerulli-Harms: University of Lausanne and London Economics Ltd, Somerset House, New Wing, London WC2R 1LA (email: a.cerulliharms@ gmail.com); Goette: University of Bonn, Institute for Applied Microeconomics, Adenauerallee 24-42, 53113 Bonn (email: lorenz.goette@uni-bonn.de); Sprenger: UC San Diego, Department of Economics, Rady School of Management, Wells Fargo Hall, 4W106 9500 Gilman Drive, La Jolla, CA 92093 (email: csprenger@ucsd.edu). We are grateful for the insightful comments of many colleagues and seminar audiences at UCSB, Caltech, Harvard University, Central European University, Stockholm School of Economics, the University of Bonn, the University of Zurich, Universitat Pompeu Fabra, Royal Holloway University of London, University of Oxford, and University of Lausanne. We also acknowledge the generous support of the Swiss National Science Foundation, grant CR11I1_137980 (Goette), and the National Science Foundation, grant SES-1024683 (Sprenger). The authors declare having no conflict of interest. This research was undertaken while Cerulli-Harms and Goette were at the Department of Economics at the University of Lausanne. Institutional Review Board approval for the collection of data on human subjects has been obtained by the Ethics Committee of the Faculty of Business and Economics, University of Lausanne where all the relevant experiments have been conducted.

${ }^{\dagger}$ Go to https://doi.org/10.1257/mic.20170271 to visit the article page for additional materials and author disclosure statement(s) or to comment in the online discussion forum.

${ }^{1}$ The KR model establishes two key innovations beyond models of disappointment aversion (Bell 1985, Loomes and Sugden 1986, and Gul 1991). First, as opposed to a certainty equivalent-based reference point, each element of the distribution of expected outcomes serves as a possible reference point. Second, the KR model establishes several key rational expectations equilibrium concepts requiring consistency between expectations and consumption outcomes in equilibrium.
} 
To facilitate these applications, KR makes the additional assumption that expectations are rational. Consider the standard exchange asymmetry of an "endowment effect" (Kahneman, Knetsch, and Thaler 1990), wherein sellers' valuations for a given object exceed buyers' valuations. A seller can either expect to sell or expect not to sell. If she expects not to sell, her reference point is keeping the object, and she forecasts her sensations of losses when asked to give up the object in exchange for money. Under these expectations, she will require compensation in excess of the object's intrinsic value to indeed relinquish it. A similar logic lowers buyers' valuations conditional on expecting not to buy. ${ }^{2}$ Both buyers and sellers, when determining a plan of action, i.e., a valuation, forecast their own sensations of gains and losses for each given reference point. If a reference point reinforces a given action (e.g., a seller expecting not to sell and indeed not selling), the action can be supported in a rational expectations equilibrium termed a Personal Equilibrium (PE) ${ }^{3}$ In the KR model, if a buyer expects not to buy and a seller expects not to sell, they can potentially support an endowment effect in PE. ${ }^{4}$

We construct standard exchange experiments with one critical innovation. We introduce a probability of forced exchange. That is, as in previous exchange experiments, individuals are endowed with objects (in our case a university mug or CHF $10 \approx$ US $\$ 10$ ). Sellers (endowed with mugs) are asked whether they are willing to sell at various prices to elicit their Willingness To Accept (WTA). Buyers (endowed with money) are asked whether they are willing to buy at various prices to elicit their Willingness To Pay (WTP). Our designs take this otherwise standard environment and introduce a probability of forced exchange. Note, WTA and WTP are elicited, but, regardless of the participants' preferences, with probability $p$ exchange will be forced at an arbitrary price. This means that mugs will be confiscated from sellers and replaced with a payment (and vice versa for buyers) with this known, fixed probability.

The probability of forced exchange affects the expectations of the sellers (and buyers): they can no longer expect to hold on to their mug (not buy a mug), and consequently, their reference point should adjust, if the personal-equilibrium prediction of the KR model is correct. With the mug probabilistically gone (bought), selling (buying) feels less like a loss. Thus, in the KR model, as the probability of forced exchange is altered, so too are attitudes toward exchange. Buyers' valuations increase and sellers' valuations decrease as the probability of forced exchange grows. Our experiments are predicated on testing this asymmetry in comparative statics. One stark example of such changing attitudes occurs with forced exchange probability of $p=0.5$. For $p=0.5$, the reference points of both buyers expecting not to buy and sellers expecting not to sell must be identical. Both must expect to have the mug with probability 0.5 or the market

\footnotetext{
${ }^{2}$ A buyer can either expect to buy or expect not to buy. If he expects not to buy, his reference point is keeping his money, and he forecasts his sensations of losses when asked to give up money in exchange for the object. Hence, if he expects not to buy, he will require a price below the object's intrinsic value to relinquish his money.

${ }^{3}$ Our implemented equilibrium concept is Personal Equilibrium (Kosszegi and Rabin 2006). In Section I, we also provide some discussion of the equilibrium refinement concepts, Preferred Personal Equilibrium and ChoiceAcclimating Personal Equilibrium, which select among a potential multiplicity of personal equilibria.

${ }^{4} \mathrm{We}$ formalize this result in Section I.
} 
price with probability 0.5 . Having identical reference points implies having identical preferences under a strict reading of the KR model. We demonstrate that with forced exchange probability 0.5 there is no PE with endowment effect exchange asymmetries in the KR model, and, beyond probability 0.5 , only PEs with reverse endowment effects exist.

We test for the effects of probabilistic forced exchange in a series of three experiments with a total of 930 subjects. Subjects are endowed with a mug or money and WTA $/ W T P$ are elicited. Across experimental conditions, $p$ is increased from 0 (no forced exchange) to $0.25,0.5,0.75$, and 0.99.5 Our experiments differ in the ordering of events and the structure of price determination. In experiment 1 , subjects are provided their endowments (of mugs or money) first, and then explained the forced exchange mechanism. In experiments 2 and 3 , forced exchange is explained first, and then endowments are provided. In experiments 1 and 2, the price determination mechanism is the standard market-price determination from Kahneman, Knetsch, and Thaler (1990). In experiment 3, a random price is drawn for each subject. ${ }^{6}$

Our results are mixed and highlight a sensitivity to small nuances in experimental design. In experiment 1 , an endowment effect exists at $p=0$ and neither buyers' nor sellers' valuations are influenced by probabilistic forced exchange. The asymmetric comparative static predictions are easily tested with a difference-in-differences analysis that generates a clear null effect. In experiment 2 , with the forced exchange mechanism presented first, the results are much more promising for the KR model, with both buyers and sellers responding to forced exchange in the predicted direction. In experiment 3 , with a random price determination mechanism, the results again are null.

The sensitivity of our results to relatively minor details outside of the model is a striking finding. Previous studies have tested different aspects of expectation-based reference dependence in the context of exchange behavior than considered here, and also documented a sensitivity of results to minute experimental detail. Ericson and Fuster (2011), Camerer et al. (2016), and Heffetz and List (2014) manipulate the probability with which subjects will be permitted to exchange or choose objects.7 While Ericson and Fuster (2011) and Camerer et al. (2016) find that lower permission probabilities increase the tendency that subjects keep their initial object,

\footnotetext{
${ }^{5}$ Our design eliminates important potential confounds outlined in the critiques of Plott and Zeiler (2005, 2007) and Ericson and Fuster (2011) for subjects inferring some specific value from their endowment. First, subjects are randomized to being buyers or sellers. Hence, if an object will be taken away from one person with a specified probability, it will be given to someone else, limiting subjects' ability to make value inferences about the objects in question. Second, subjects are forced to consider the alternative object even if they hope to keep their endowment because, with a fixed probability, the alternative object will be theirs.

${ }^{6}$ This eliminates the noted possibility that buyers and sellers may have had differing price expectations (which could affect reservation prices in KR), and the possibility that buyers and sellers mistakenly believe they have market power (Plott and Zeiler 2007).

${ }^{7}$ There is a key distinction between our analysis of probabilistic forced exchange and this probabilistic permission to exchange or choose. In Section I, we show two important facts about such experiments. First, not responding to the permission probability is fully compatible with PE. Second, only individuals who wish to give up their initial object can be affected by this experimental manipulation, but these individuals would fail to deliver an endowment effect to begin with. By contrast, our manipulation should act on those individuals who would not wish to exchange and delivers testable comparative statics when unwillingness to exchange is driven (at least partially) by PE considerations.
} 
Heffetz and List (2014) do not find a significant impact of the same manipulation on choice probabilities in a substantially larger sample. ${ }^{8} \mathrm{~A}$ fair reading of these findings would likely indicate that establishing expectations-based reference points is a challenging endeavor, with other decision elements having the potential to deeply influence behavior. This point is furthered by our general rejection of the strict point predictions of the KR model. Though under a strict reading of the KR model, there can be no endowment effect with $p>0.5$, an endowment effect persists in all of our experimental conditions. This suggests additional forces or points of reference driving a hesitance towards exchange.

The remainder of the paper is structured as follows. Section I lays out the basic framework and derives our test of comparative statics. Section II presents the design of our experiments. Section III presents results, and Section IV concludes.

\section{Theory}

The KR model establishes a reference-dependent utility function, $U(F \mid G)$, which evaluates a distribution of consumption outcomes, $F$, in comparison to a distribution of possible reference points, $G$. The outcomes of $F$ are consumption vectors, $\mathbf{c}$, while the outcomes of $G$ are reference point vectors, $\mathbf{r}$. The KR utility is written as

$$
U(F \mid G)=\iint u(\mathbf{c} \mid \mathbf{r}) d F(\mathbf{c}) d G(\mathbf{r}) .
$$

For our purposes, we consider utility over two dimensions: mugs, $m$, and money, $y$, such that $\mathbf{c}=(m, y)$ and $\mathbf{r}=\left(r_{m}, r_{y}\right)$. The value $m \in\{0, M\}$ indicates the consumption utility of having no mug or one mug and $y$ is the utility from income available for other consumption. The values $r_{m}$ and $r_{y}$ correspond to the reference utility outcomes for mugs and money, respectively. ${ }^{9}$ Following Kőszegi and Rabin (2006), we assume separability across dimensions and a specific functional form of reference dependence applied equally across dimensions ${ }^{10}$ such that

$$
u(\mathbf{c} \mid \mathbf{r})=u\left(m, y \mid r_{m}, r_{y}\right)=m+y+\mu\left(m-r_{m}\right)+\mu\left(y-r_{y}\right),
$$

where

$$
\mu(z)= \begin{cases}\eta z & \text { if } z \geq 0 \\ \eta \lambda z & \text { if } z<0\end{cases}
$$

\footnotetext{
${ }^{8}$ Heffetz and List (2014) differentiates between the Ericson and Fuster (2011) protocol of endowing subjects with objects and probabilistically permitting them to exchange and their own protocol, where subjects are told that with only a given probability will they be allowed to choose which object to receive (and otherwise be given one of the objects previously determined at random).

${ }^{9}$ This structure assumes linear utility for income because we consider only small changes in endowments with expected negligible income effects.

${ }^{10}$ The assumption of a global gain-loss function is made for both tractability and discipline in Kôszegi and Rabin (2006). In our context, it delivers clear symmetries in expected behavior across conditions. The comparative statics, however, would be maintained if one allowed the shape of the gain-loss function to vary across goods.
} 
The parameter $\eta$ represents the value of changes from the reference point and $\lambda$ corresponds to the degree of loss aversion. ${ }^{11}$

With these preferences in hand, Kószegi and Rabin (2006) ensure the consistency of choice through the notion of personal equilibrium. Consider a choice set, $\mathcal{D}$, composed of lotteries, $F$, over consumption outcomes $\mathbf{c}=(m, y)$.

DEFINITION 1 (Personal Equilibrium $(P E)$ ): A choice $F \in \mathcal{F}$, is a personal equilibrium if

$$
U(F \mid F) \geq U\left(F^{\prime} \mid F\right) \quad \forall F^{\prime} \in \mathcal{D} .
$$

When considering $F$ as the reference distribution, the decision maker prefers to consume $F$ as a consumption distribution over any other consumption distribution $F^{\prime}$. The coincidence of the reference and consumption distributions in a PE provides a sense in which the model follows rational expectations. In a PE, the decision maker can only expect as the reference point something he will consume given that he expects it.

Figure 1 illustrates predictions of the KR model under probabilistic forced exchange with parameters $\eta=1, \lambda=2$, and the consumption utility of a mug, $M$, normalized to 1 . Buyers and sellers are told that with probability, $p$, exchange will be forced. Mugs will be confiscated from sellers, a price $x$ will be confiscated from buyers, and transactions will occur. Under KR, probabilistic forced exchange generates a stochastic reference point for market participants wishing not to exchange. Buyers and sellers wishing not to exchange must hold as possible reference points both prospects, mug, and money, each with a certain probability. As these probabilities shift, so too do attitudes toward exchange.

Consider a seller expecting not to sell facing forced exchange probability, $p$, at a given price, $x$. She now holds the reference distribution under forced exchange, $G\left(r_{m}, r_{y}\right)=p \cdot(0, x)+(1-p) \cdot(M, 0)$. She contemplates keeping her mug, inducing the consumption distribution $F(m, y)=p \cdot(0, x)+(1-p) \cdot(M, 0)$; or selling her mug, inducing the degenerate distribution $F(m, y)=1 \cdot(0, x)$. She can support not selling, and trying to keep her mug in PE, provided

$$
\begin{gathered}
U(p \cdot(0, x)+(1-p) \cdot(M, 0) \mid p \cdot(0, x)+(1-p) \cdot(M, 0)) \\
\geq U(1 \cdot(0, x) \mid p \cdot(0, x)+(1-p) \cdot(M, 0))
\end{gathered}
$$

\footnotetext{
${ }^{11}$ For ease of explication, we primarily discuss this piecewise linear gain-loss function. Importantly, the central personal equilibrium predictions and comparative statics are maintained when we consider more general functional forms. Appendix sections A and B provide the details.
} 


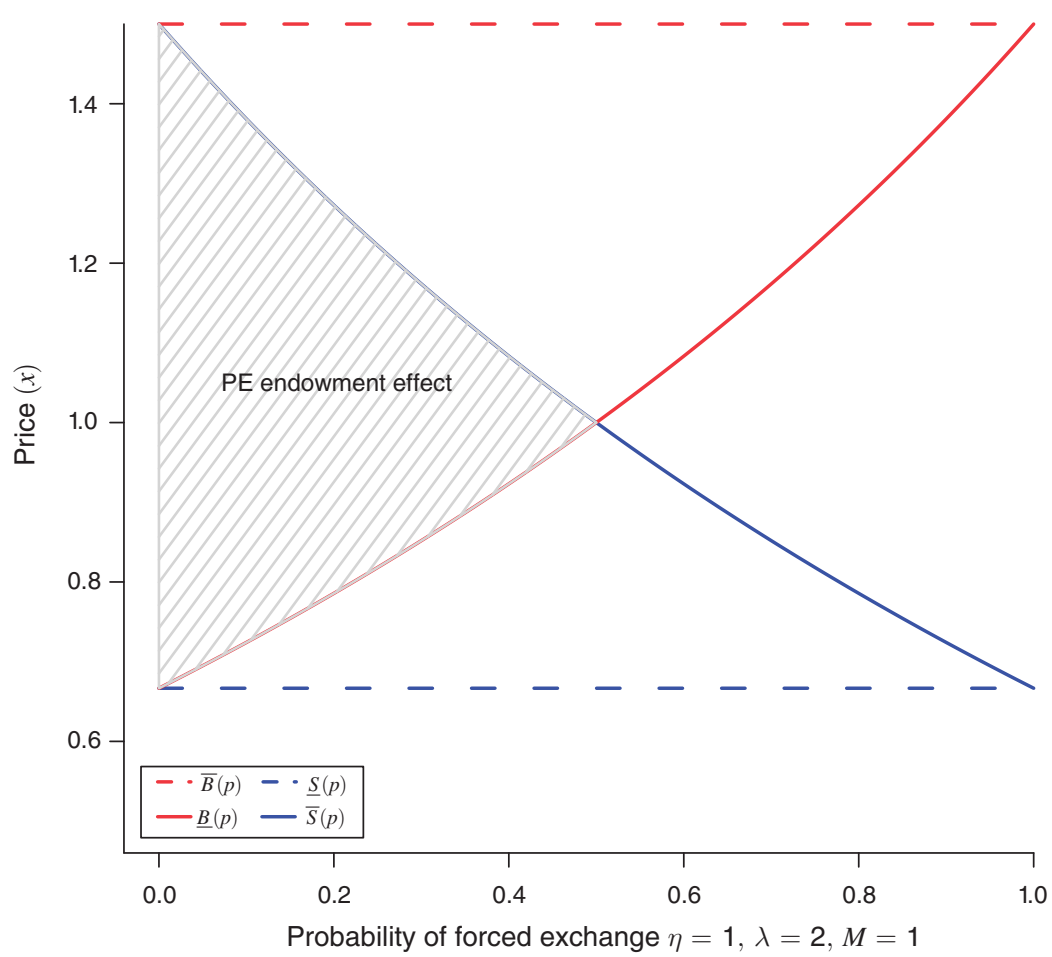

Figure 1. Personal Equilibria and Endowment EfFects

Notes: Solid lines correspond to $\underline{B}(p)$, the lowest price at which the buyer can support not buying (red), and $\bar{S}(p)$, the highest price at which the seller can support not selling (blue). Dashed lines correspond to $\bar{B}(p)$, the highest price at which the buyer can support buying (red), and $\underline{S}(p)$, the lowest price at which the seller can support selling (blue). Gray shaded area corresponds to prices at which the buyer can support not buying and the seller can support not selling, i.e., where a PE endowment effect can exist.

or

$p x+(1-p) M+p(1-p) \eta(1-\lambda)(M+x) \geq x+(1-p) \eta(x-\lambda M) \cdot 12$

${ }^{12}$ Specifically, the utility values are

$$
\begin{aligned}
& U(p \cdot(0, x)+(1-p) \cdot(M, 0) \mid p \cdot(0, x)+(1-p) \cdot(M, 0)) \\
&= p[x+(1-p)\{\eta \lambda(0-M)+\eta(x-0)\}+p\{\eta \lambda(0-0)+\eta \lambda(x-x)\}] \\
& \quad+(1-p)[M+(1-p)\{\eta \lambda(M-M)+\eta \lambda(0-0)\}+p\{\eta(M-0)+\eta \lambda(0-x)\}] \\
&= p x+(1-p) M+p(1-p) \eta(1-\lambda)(M+x),
\end{aligned}
$$

and

$$
\begin{aligned}
& U(1 \cdot(0, x) \mid p \cdot(0, x)+(1-p) \cdot(M, 0)) \\
& \quad=x+(1-p)\{\eta \lambda(0-M)+\eta(x-0)\}+p\{\eta \lambda(0-0)+\eta \lambda(x-x)\} \\
& \quad=x+(1-p) \eta(x-\lambda M) .
\end{aligned}
$$


Solve for the highest price at which the seller can support not selling the mug in PE as

$$
\bar{S}(p)=\frac{(1+p(1-\lambda) \eta+\eta \lambda)}{(1-p(1-\lambda) \eta+\eta)} M
$$

The function $\bar{S}(p)$ is graphed as the solid blue line in Figure 1. The important insight is that in the interval of forced exchange $p \in[0,1], \bar{S}(p)$ is strictly decreasing for $\eta>0$ and $\lambda>1$. The maximum price at which a seller can support not selling the mug is decreasing as one increases the forced exchange probability.

Also graphed in Figure 1 is

$$
\underline{S}(p)=\frac{(1+\eta)}{1+\eta \lambda} M,
$$

the lowest price at which the seller can support selling. ${ }^{13}$ Note that $\underline{S}(p)$ is constant with respect to $p$; because the seller is giving up the object, she will not face any gains or losses related to $p$.

Hence, as $p$ increases, the region $(\underline{S}(p), \bar{S}(p)]$ shrinks, and it becomes more difficult to find transaction prices, $x$, at which the seller can support not selling in a PE. The seller's attitudes toward exchange grow more favorable the more likely it is that she is forced to exchange.

Next, consider a buyer expecting not to exchange facing forced exchange probability, $p$, at a given price, $x$. He now holds as the reference distribution under forced exchange, $G\left(r_{m}, r_{y}\right)=p \cdot(M, 0)+(1-p) \cdot(0, x)$. He contemplates keeping his money, inducing the consumption distribution $F(m, y)=p \cdot(M, 0)+(1-p) \cdot(0, x)$, or purchasing the mug, inducing the degenerate distribution $F(m, y)=1 \cdot(M, 0)$. He can support not buying and trying to keep his money in PE provided

$$
\begin{aligned}
& U(p \cdot(M, 0)+(1-p) \cdot(0, x) \mid p \cdot(M, 0)+(1-p) \cdot(0, x)) \\
& \quad \geq U(1 \cdot(M, 0) \mid p \cdot(M, 0)+(1-p) \cdot(0, x)),
\end{aligned}
$$

\footnotetext{
${ }^{13}$ Consider a seller who expects to sell under probabilistic forced exchange. She holds as reference distribution $\left(r_{m}, r_{y}\right)=(0, x)$. She can support selling her object in PE provided

or

$$
u(0, x \mid 0, x) \geq U(p \cdot(0, x)+(1-p) \cdot(M, 0) \mid 0, x)
$$

$$
x \geq p x+(1-p)[M+\eta(M-0)+\eta \lambda(0-x)] .
$$
}

Solve for the lowest price at which the seller can support selling her object as

$$
\underline{S}(p)=\frac{(1+\eta)}{1+\eta \lambda} M .
$$


or

$$
p M+(1-p) x+p(1-p) \eta(1-\lambda)(M+x) \geq M+(1-p) \eta(M-\lambda x) \cdot 14
$$

Solve for the lowest price at which the buyer can support not buying the mug in PE as

$$
\underline{B}(p)=\frac{(1-p(1-\lambda) \eta+\eta)}{1+p(1-\lambda) \eta+\eta \lambda} M .
$$

The function $\underline{B}(p)$ is graphed as the solid red line in Figure 1. Note that in the interval of forced exchange $p \in[0,1], \underline{B}(p)$ is strictly increasing for $\eta>0$ and $\lambda>1$. The minimum price at which a buyer can support not buying is increasing as one increases the forced exchange probability.

Also graphed in Figure 1 is

$$
\bar{B}(p)=\frac{(1+\eta \lambda)}{1+\eta} M,
$$

the highest price at which the buyer can support buying. ${ }^{15}$ Note that $\bar{B}(p)$ is, again, constant with respect to $p$.

As $p$ increases, the region $(\underline{B}(p), \bar{B}(p)]$ shrinks and it becomes more difficult to find transaction prices, $x$, at which the buyer can support not buying in a PE.

$$
\begin{aligned}
& { }^{14} \text { Specifically, the utility values are } \\
& \begin{aligned}
& U(p \cdot(M, 0)+(1-p) \cdot(0, x) \mid p \cdot(M, 0)+(1-p) \cdot(0, x)) \\
&= p[M+(1-p)\{\eta \lambda(0-M)+\eta(x-0)\}+p\{\eta \lambda(0-0)+\eta \lambda(x-x)\}] \\
& \quad+(1-p)[x+(1-p)\{\eta \lambda(M-M)+\eta \lambda(0-0)\}+p\{\eta(M-0)+\eta \lambda(0-x)\}] \\
&= p M+(1-p) x+p(1-p) \eta(1-\lambda)(M+x),
\end{aligned}
\end{aligned}
$$

and

$$
\begin{aligned}
U(1 & \cdot(M, 0) \mid p \cdot(M, 0)+(1-p) \cdot(0, x)) \\
& =M+(1-p)\{\eta(M-0)+\eta \lambda(0-x)\}+p\{\eta \lambda(M-M)+\eta \lambda(0-0)\} \\
& =M+(1-p) \eta(M-\lambda x) .
\end{aligned}
$$

${ }^{15}$ Consider a buyer who expects to buy under probabilistic forced exchange. She holds as reference distribution $\left(r_{m}, r_{y}\right)=(M, 0)$. He can support buying in PE provided

or

$$
u(M, 0 \mid M, 0) \geq U(p \cdot(M, 0)+(1-p) \cdot(0, x) \mid M, 0)
$$

$$
M \geq p M+(1-p)[x+\eta(x-0)+\eta \lambda(0-M)] .
$$

Solve for the highest price at which the buyer can support buying as

$$
\bar{B}(p)=\frac{(1+\eta \lambda)}{1+\eta} M
$$


The buyer's attitudes towards exchange grow more favorable the more likely it is she is forced to exchange.

Figure 1 also illustrates the way in which the KR model can support an endowment effect in PE. The shaded gray area identifies prices at which it is PE for buyers to not buy and it is PE for sellers to not sell. The range of such prices shrinks as $p$ increases and is eliminated entirely for $p>0.5$.

Transitioning from our theoretical development to predictions for individual behavior requires a mapping from PE choices to statements on buyer and seller valuations. We make a first assumption that for all prices greater than $\underline{B}(p)$, the buyer chooses not to buy, following the PE most conducive to generating endowment effects. At prices below $\underline{B}(p)$, we assume the buyer chooses to buy, such that $\underline{B}(p)$ is equivalent to Willingness To $\operatorname{Pay,} \operatorname{WTP}(p)$.

Similarly, we assume that for all prices less than $\bar{S}(p)$, the seller chooses not to sell, following the PE most conducive to generating endowment effects. At prices above $\bar{S}(p)$, we assume the seller chooses to sell, such that $\bar{S}(p)$ is equivalent to Willingness To Accept, $W T A(p) .{ }^{16}$ This mapping in hand, we draw the following implication:

Comparative Static Asymmetry: $W T P(p)$ increases and $W T A(p)$ decreases as $p$ increases.

It is important to note that the asymmetry of reactions to probabilistic forced exchange is not the only potentially testable prediction. The KR model also makes a variety of point predictions. First, the KR model predicts that at $p=0.5$ there exists no PE with an endowment effect as $\bar{S}(0.5)=\underline{B}(0.5)$; the highest price at which the seller can support not selling in PE is exactly equal to the lowest price the buyer can support not buying. For $p>0.5$ only PE with reverse endowment effects exist as $\bar{S}(p)>\underline{B}(p)$ for $p>0.5$. Additionally, a symmetry in valuations should exist as $\bar{S}(p)=\underline{B}(1-p)$. These predictions rely on a strict reading of the $\mathrm{KR}$ model, that behavior is driven exclusively by PE considerations. As such, they may not hold if other forces beyond those of PE influence valuations.

Two issues present relevant threats to testing the KR model's point and general predictions in our setting. First, the development above indicates that for a given price, as the forced exchange probability increases, it will become less likely that not exchanging can be supported in PE. However, it may well be that the selected PE generates exchange in the first place (i.e., buyers expect to buy and support doing so, sellers expect to sell and support doing so), or that the equilibrium selection process is influenced by the forced exchange probability (i.e., the selected equilibria move from being anti-exchange to pro-exchange as $p$ increases). In such cases we may find a lack of sensitivity to changing probabilities.

\footnotetext{
${ }^{16}$ In the Mathematical Appendix, Section D, we relax the assumption that WTA and WTP identify $\bar{S}(p)$ and $\underline{B}$ $(p)$, respectively. We analyze explicitly how choices from a list of prices, where each price could be chosen with a certain probability, interacts with our mechanism. Intuitively, a price list weakens the endowment effect, as sellers have to expect to sell at very high prices, and buyers have to expect to buy at very low prices.
} 
Importantly, the KR model is developed with notions of equilibrium refinement, which provide structured processes for equilibrium selection. We consider the refinements termed Preferred Personal Equilibrium (PPE) and Choice-Acclimating Personal Equilibrium (CPE). Take a choice set, $\mathcal{D}$, composed of lotteries, $F$, over consumption outcomes $\mathbf{c}=(m, y)$. Let $P E(\mathcal{D})$ be the set of Personal Equilbria from choice set $\mathcal{D}$.

DEFINITION 2 (Preferred Personal Equilibrium $(P P E)$ ): A choice $F \in P E(\mathcal{D})$ is a preferred personal equilibrium if

$$
U(F \mid F) \geq U\left(F^{\prime} \mid F^{\prime}\right) \quad \forall F^{\prime} \in P E(\mathcal{D}) .
$$

DEFINITION 3 (Choice-Acclimating Personal Equilibrium (CPE)): A choice $F \in \mathcal{D}$ is a choice-acclimating personal equilibrium if

$$
U(F \mid F) \geq U\left(F^{\prime} \mid F^{\prime}\right) \quad \forall F^{\prime} \in \mathcal{D} \text {. }
$$

PPE compares ex ante utility values for PE choices to select among a potential multiplicity of equilibria. CPE compares ex ante utility values for all options in $\mathcal{D}$ without restriction to the set $P E(\mathcal{D})$.

Though the point predictions for behavior break down, under both the PPE and CPE refinements the qualitative effects of forced exchange probability persist. As $p$ increases, the set of prices at which buyers choose to buy and sellers choose to sell grows. Figure 2, panel A presents the PPE and CPE cutoff values for buying and selling prices under the parameters $\eta=1$, and $\lambda=2$, normalized by the consumption value, $M=1$.

The implication that attitudes toward exchange improve as $p$ increases is maintained under CPE and PPE and can take an even more drastic form. Indeed, the endowment effect itself does not survive the CPE and PPE refinements. ${ }^{17}$ These already positive attitudes toward exchange are made even more so by forced exchange. ${ }^{18}$ Mathematical Appendix, Section C provides mathematical detail.

Second, a discussion has developed around experimental methodologies that enhance or reduce the endowment effect (Plott and Zeiler 2005, 2007). Forces such as viewing the endowed object as a gift or believing that one has market power may potentially influence the extent to which buyers express different valuations than sellers. We view these forces as systematically changing intrinsic valuations for the mug, $M$, between buyers and sellers. Hence, the regions in which buyers and sellers can support exchanging or not are systematically shifted. The intrinsic value of sellers for the object may exceed that of buyers. In Figure 2, panel B we present

\footnotetext{
${ }^{17}$ Consider the possibility that a buyer holding on to his money, $(0, x)$, and a seller holding on to her mug, $(M, 0)$, are both supportable PE. The PPE refinement compares the utility values, $u(0, x \mid 0, x)$ and $u(M, 0 \mid M, 0)$. The CPE refinement compares these same values without the restriction that both actions are PE. Absent the pathological case of equality, one must yield a higher valuation and be selected as the refined choice by both buyers and sellers.

${ }^{18}$ Exchanging assures no unexpected losses under probabilistic forced exchange and so is preferred to not exchanging even at quite extreme prices in CPE.
} 
Panel A. Equilibrium refinement

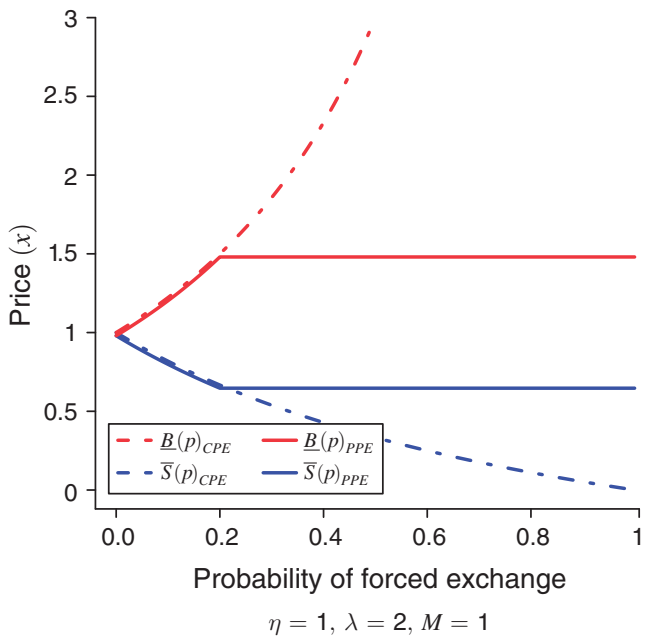

Panel B. Shifted valuations

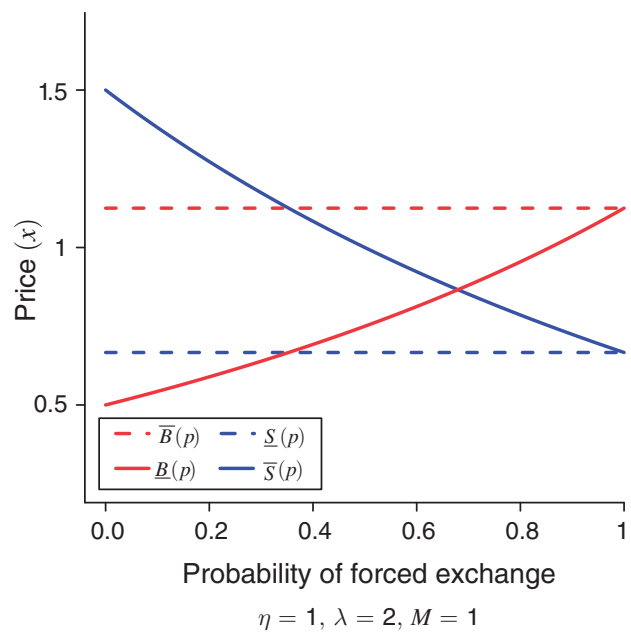

Figure 2. EQuilibrium Refinement and SHifted VALUATIONS

Notes: Panel A: solid lines correspond to $\underline{B}(p)_{P P E}$, lowest price at which the buyer can support not buying in PPE(red), and $\bar{S}(p)_{P P E}$, highest price at which the seller can support not selling in PPE (blue). Dashed lines correspond to $\underline{B}(p)_{C P E}$, lowest price at which the buyer can support not buying in CPE (red), and $\bar{S}(p)_{C P E}$, highest price at which the seller can support not selling (blue). The horizontal portion of $\underline{B}(p)_{P P E}$ corresponds to $\bar{B}(p)$. At prices above $\bar{B}(p)$ there is no PE in which the buyer buys the object and so the only remaining PE entails the buyer not buying, leading PPE and CPE to different predictions. The horizontal portion of $\bar{S}(p)_{P P E}$ corresponds to $\underline{S}(p)$ following a similar logic. Panel B: shifted valuations moves relevant cones, distorting the probabilities at which a PE endowment effect maintains.

such a shift. Though point predictions again break down, the general implication on attitudes toward exchange should be maintained. If reluctance to exchange is at least partially driven by PE considerations, it should be influenced by the probability of forced exchange. 19

The theoretical prediction tested here differs from those examined in earlier work. Ericson and Fuster (2011); Camerer et al. (2016), and Heffetz and List (2014) all consider probabilistic permission to exchange or choose. For example, Ericson and Fuster (2011) endow subjects with an object, a university pen, and examine whether they are willing to exchange for another object, a university mug, under varying probability of exchange permission. Individuals holding a reference point of keeping their object (that is, those individuals that deliver the endowment effect in KR) are unaffected by the permission to trade. ${ }^{20}$ Further, individuals who have sufficiently

\footnotetext{
${ }^{19}$ A natural extension of the argument above is that changes to forced exchange probability induce changes to valuations. For example, higher forced exchange probability could make buyers feel the object is less valuable and sellers that the object is more valuable. Our experimental design limits the plausibility of such effects as all of our experimental markets were conducted simultaneously with random assignment to forced exchange probability.

${ }^{20}$ In a mug-pen exchange environment, let $u_{p}$ be the consumption utility of a pen and $u_{m}$ be the consumption utility of a mug. Under probabilistic permission of exchange, $q$, a person endowed with a pen may hold as a
} 
strong preferences for the mug instead of the pen, can exchange in PE at any probability and so may also be unaffected by the manipulation. ${ }^{21}$ Importantly, the set of relative mug-pen valuations required for supporting exchange at any probability is the complement of the set of relative mug-pen valuations for supporting keeping the pen. Stated differently, if an individual cannot support keeping his object in PE, he can support exchanging at any probability. Not responding to the permission to exchange is thus fully consistent with PE. In our design, if the prominent finding of an endowment effect is driven at least partially by PE, PPE, or CPE considerations, willingness to exchange must increase with the probability of forced exchange.

\section{Experimental Setup}

We conducted three exchange experiments at the University of Lausanne with a total of 930 subjects. Experiment 1 was conducted in a large first year introductory economics class following closely the setup of Kahneman, Knetsch, and Thaler (1990), but with a probabilistic forced exchange. Four-hundred and sixty five student subjects were separated by the middle aisle of a large, symmetric classroom into a group of buyers $(N=236)$, sitting to the left, and a group of sellers $(N=229)$, sitting to the right of the aisle. ${ }^{22}$

All students were given a package of instructions and, at the same time, every student in the group of sellers had a university mug placed on the desk in front of them. The instructions described the content, tasks, and payments of the experiment. Sellers (buyers) were told that they were now in possession of a university mug (CHF $10 \approx$ US\$10), which they could potentially sell (use to buy a mug). Participants were described the market price determination mechanism and were

reference point either keeping the pen $\left(u_{p}, 0\right)$ or exchanging when permitted to do so $q \cdot\left(0, u_{m}\right)+(1-q) \cdot\left(u_{p}, 0\right)$. The PE supporting keeping the object is unaffected by $q$. To see this, note

$$
\begin{aligned}
U\left(u_{p}, 0 \mid u_{p}, 0\right) & \geq U\left(q \cdot\left(0, u_{m}\right)+(1-q) \cdot\left(u_{p}, 0\right) \mid u_{p}, 0\right) \Rightarrow \\
u_{p} & \geq q \cdot\left[u_{m}+\eta\left(u_{m}-0\right)+\eta \lambda\left(0-u_{p}\right)\right]+(1-q) \cdot u_{p} \Rightarrow \\
u_{p} & \geq\left[u_{m}+\eta\left(u_{m}-0\right)+\eta \lambda\left(0-u_{p}\right)\right] \Rightarrow \\
u_{p} & \geq \frac{1+\eta}{1+\eta \lambda} u_{m}
\end{aligned}
$$

is independent of the probability, $q$.

21 The individual can support attempting to exchange in PE, for $\lambda>1$, provided

$$
\begin{aligned}
U\left(q \cdot\left(0, u_{m}\right)+(1-q) \cdot\left(u_{p}, 0\right) \mid q \cdot\left(0, u_{m}\right)+(1-q) \cdot\left(u_{p}, 0\right)\right) & \geq U\left(u_{p}, 0 \mid q \cdot\left(0, u_{m}\right)+(1-q) \cdot\left(u_{p}, 0\right)\right) \Rightarrow \\
q u_{m}+(1-q) u_{p}+q(1-q) \eta(1-\lambda)\left(u_{m}+u_{p}\right) & \geq u_{p}+q\left[\eta u_{p}-\eta \lambda u_{m}\right] \Rightarrow \\
q & \geq \frac{(1+\eta \lambda) u_{p}-(1+\eta) u_{m}}{\eta(\lambda-1)\left(u_{m}+u_{p}\right)} .
\end{aligned}
$$

Note that if $u_{p} \leq \frac{1+\eta}{1+\eta \lambda} u_{m}$, exchange is supportable for all $q \in[0,1]$ in PE, and if $u_{p} \geq \frac{1+\eta \lambda}{1+\eta} u_{m}$, exchange is not supportable for any $q \in[0,1]$. When combined with the above equilibrium condition on not exchanging, this development implies that if an individual cannot support keeping his object in PE, $u_{p}<\frac{1+\eta}{1+\eta \lambda} u_{m}$, he can support exchanging at any probability.

${ }^{22}$ We have no prior reason to believe that people sitting on one versus the other side would have different consumption values for the traded objects or different levels of reference-dependence. 
exhorted to reveal their true valuations. ${ }^{23}$ The online Appendix provides translated instructions.

Subjects were assigned at random to one of four different forced exchange probabilities, $p \in\{0,0.25,0.5,0.75\}$, by providing them with a different packet of instructions. Within the group of either buyers and sellers, we randomized probability conditions by changing the instructions after every sixth individual. Hence, all four markets were conducted at the same time. The online Appendix provides the exact seating plan of experiment 1 . Subjects were told that upon realization of the market price, a random number between 1 and 100 would be drawn. If this number was between 1 and $p$, they would be forced to sell (buy) a mug at the market price, irrespective of their responses. If the forced exchange occurred, they would receive the market price in exchange for their mug (receive a mug and pay the market price).

Once the price determination mechanism and forced exchange probability had been explained, participants indicated at which price they would be willing to sell (buy) a mug on a separate page listing prices from $\mathrm{CHF} 0.50$ to $\mathrm{CHF} 10$ in $\mathrm{CHF} 0.50$ increments. We code $\operatorname{WTP}(p)$ as the highest price for which a buyer is willing to purchase a mug before switching to preferring to keep his money. Respectively, we code $W T A(p)$ as the lowest price for which a seller prefers selling her mug before switching to preferring to keep it. ${ }^{24}$

A total of 40 students from higher years assisted the implementation of the experiment. Once all questionnaires were completed, the assistants entered all answers into a spreadsheet in order to compute market prices and to prepare payments. In the meantime, the usual lecture was held in the classroom. Shortly before the end of the lecture all participants received payments and mugs according to the outcome of the experiment. 25

Experiments 2 and 3 follow very similar designs with modifications to the ordering of events and to the price determination mechanism. In experiment 2 the design and implementation coincides exactly with experiment 1 , except that we first distributed the instructions to the participants explaining probabilistic forced exchange without showing or mentioning the mug. ${ }^{26}$ Sellers' instructions stated that they were going to receive an object and they would need to indicate their willingness to accept. For buyers the instructions said that they had ten CHF and they would need to indicate their willingness to pay for the object that the other participants were going to receive. As the purpose was to draw attention to the

\footnotetext{
${ }^{23}$ Any potential concerns about mistaken market power or subjects' beliefs about the other side of the market are alleviated by our close replication of these initial results with subjects facing a random price mechanism. See the description of experiments 2 and 3 below.

${ }^{24}$ For analytical convenience, WTA for sellers unwilling to sell for any of the possible prices is coded as CHF 10.5. We address truncation in the online Appendix.

${ }^{25}$ An anonymous identification sheet was handed with the instructions to ensure the correct attribution of payments and mugs.

${ }^{26}$ Though prima facie this seems a minor detail, it may indeed influence behavior as individuals may focus their attention disproportionately on the element that is presented first. Indeed, Köszegi and Rabin $(2006,1141)$ intuited such malleability of the reference point: "Specifically, a person's reference point is her probabilistic beliefs about the relevant consumption outcome held between the time she first focused on the decision determining the outcome and shortly before consumption occurs." Sprenger (2015) provides evidence of such "first-focus" referents for the KR model in the context of risky choice.
} 
probabilistic forced exchange mechanism, we conducted only the markets with $p \in\{0.25,0.5,0.75\}$, leaving out the baseline condition of $p=0$. The remainder of the experiment was unchanged. The online Appendix provides translated instructions. The participants in experiment 2 were 228 first-year law students at the University of Lausanne, with 118 (110) serving as sellers (buyers).

Experiment 3 again explained probabilistic forced exchange first, but substituted the market price determination with a random price determination. ${ }^{27}$ The text that previously explained the market price formation was replaced with text reflecting that the price for the object would be determined at random between CHF 0.50 and CHF 10. We conducted markets with $p \in\{0.25,0.5,0.75,0.99\}$, adding a condition with forced exchange probability of $p=0.99$ to verify behavior at the extremes of forced exchange. Participants for experiment 3 were recruited from the ORSEE participant pool for experiments at the University of Lausanne and the Ecole Polytechnique of Lausanne (Greiner 2004). We ran 17 lab sessions with a total of 237 participants, 118 (119) of whom served as sellers (buyers). The online Appendix provides translated instructions and the seat plan of an example laboratory session.

In addition to these three experiments we conducted a comprehension study with 41 subjects from the same University of Lausanne and Ecole Polytechnique of Lausanne subject pool. We inquired in detail about individuals' understanding of the experimental procedures (i.e., forced exchange and WTA/WTP elicitations) and generally about their comprehension of different probabilities. Around 85 percent of subjects correctly answered all comprehension questions, giving confidence that subjects are likely to understand the implemented protocols. The translated instructions are in the online Appendix. ${ }^{28}$

\section{Results}

Figure 3 and Table 1 present mean valuations of WTA and WTP across our three experiments with corresponding standard errors and test statistics. ${ }^{29}$ In experiment 1 , we find an initial endowment effect at $p=0$ of CHF 2.51 (standard error $=0.44)$. The difference between the two groups' average valuations is significant, $t=5.71,(p<0.01)$, and a Mann-Whitney test also rejects the null hypothesis of equal distributions, $z=5.31,(p<0.01)$. This first result confirms the usual finding in the endowment effect literature (Knetsch and Sinden

\footnotetext{
${ }^{27}$ It has been argued that differences in price expectations between buyers and sellers can influence their valuation in exchange experiments (Mazar, Kôszegi, and Ariely 2014). Another issue discussed in the endowment effect literature is the possibility that individuals may mistakenly try to exert market power despite being price takers (Plott and Zeiler 2007). The random price determination serves to overcome both of these potential issues, which could potentially drive exchange anomalies.

${ }^{28}$ To make the experience concrete, subjects in the comprehension study also faced choices, as in experiment 3 , with $p=0.5$. Twenty-one subjects were buyers and 20 subjects were sellers. As with our other experiments described below, at $p=0.5$ a substantial endowment effect exists with a mean WTP of CHF 2.81 (standard error $=0.54)$, a mean WTA of CHF $4.18(0.49)$, and an endowment effect WTA - WTP of CHF 1.36 (0.38), $(t=1.85, p=0.07)$. Given there is no experimental variation in $p$ in our comprehension study, no further discussion of the results is provided.

${ }^{29}$ The full distributions of responses in each experiment are provided in the online Appendix.
} 
Panel A. Experiment 1

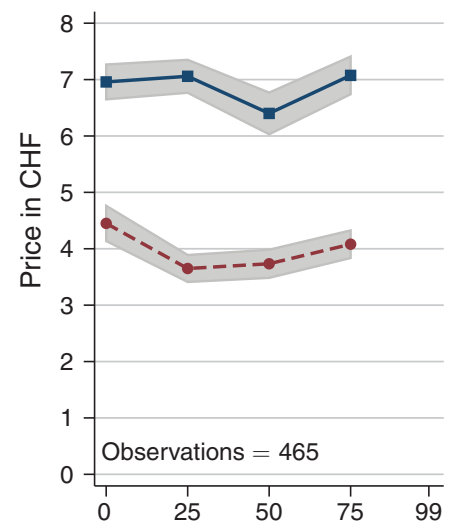

Panel B. Experiment 2

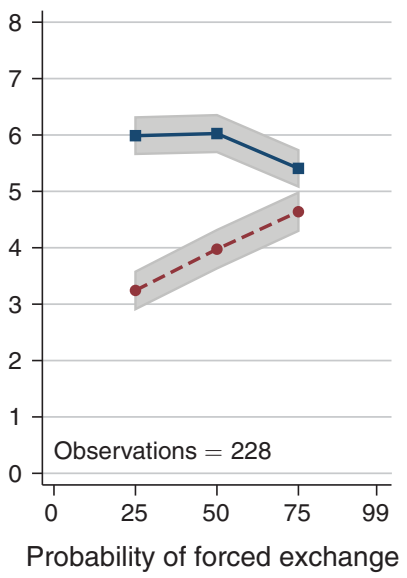

Panel C. Experiment 3

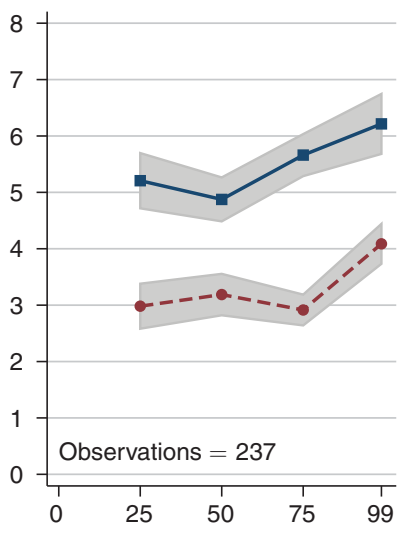

Figure 3. Experimental Valuations

1984; Knetsch 1989; Kahneman, Knetsch, and Thaler 1990), where sellers' WTA consistently exceeds buyers' WTP.

The initial unwillingness to trade in experiment 1 is virtually unaffected by probabilistic forced exchange. Sellers slightly increase their WTA and buyers slightly decrease their WTP as $p$ increases from 0 to 0.75 . Neither difference is significant and the difference-in-differences of CHF 0.49 (0.61) cannot be statistically distinguished from 0 . The KR model's asymmetric comparative static would predict decreasing WTA, increasing WTP, and a negative difference-in-differences. These predictions are not borne out in the data of experiment 1.

Experiment 2, which altered the order of the experimental instructions to explain probabilistic forced exchange, provides a qualitatively different picture of behavior. Sellers' WTA reduce by CHF $0.58(0.46),(t=1.27, p=0.21)$, and buyers' WTP increase by CHF $1.40(0.48),(t=2.94, p<0.01)$ as $p$ increased from 0.25 to 0.75 . The difference-in-differences of CHF $-1.98(0.66)$ is significant at all conventional levels, $t=2.99,(p<0.01)$. Attitudes toward exchange are substantially improved by probabilistic forced exchange, reducing the magnitude of the endowment effect by 72 percent $(-1.98 / 2.74)$ as $p$ increases from 0.25 to 0.75 . Though the point predictions of the KR theory would still be rejected (e.g., a substantial endowment effect exists at $p=0.5$ ), the model's asymmetric comparative static predictions are clearly supported.

Experiment 3, which also first explained probabilistic forced exchange but implemented a random price determination mechanism, demonstrates the fragility of the positive results of experiment 2. Consistent with the KR model, Buyers' WTP increases by $1.11(0.64),(t=1.73, p=0.09)$, as $p$ increases from $p=0.25$ to $p=0.99$. However, in contrast to the KR model, sellers' WTA also increases by a similar magnitude, CHF $1.01(0.65),(t=1.56, p=0.12)$. As such, the difference-in-differences is indistinguishable from 0 , with limited change in the 
TABLE 1 -VALUATIONS IN EXPERIMENTS

\begin{tabular}{|c|c|c|c|c|c|c|c|}
\hline Forced exchange probability & $\begin{array}{l}\text { Sellers } \\
(\#)\end{array}$ & $\begin{array}{c}\text { Buyers } \\
(\#)\end{array}$ & $\begin{array}{l}\text { WTA } \\
(\mathrm{SE})\end{array}$ & $\begin{array}{l}\text { WTP } \\
(\mathrm{SE})\end{array}$ & $\begin{array}{l}\text { WTA-WTP } \\
\quad(\mathrm{SE})\end{array}$ & $\begin{array}{c}t \text {-test } \\
(p \text {-value })\end{array}$ & $\begin{array}{l}\text { MW-test } \\
\text { ( } p \text {-value })\end{array}$ \\
\hline $\begin{array}{l}\text { Panel A. Experiment } 1 \\
p=0\end{array}$ & 60 & 60 & $\begin{array}{c}6.96 \\
(0.31)\end{array}$ & $\begin{array}{l}4.45 \\
(0.31)\end{array}$ & $\begin{array}{c}2.51 \\
(0.44)\end{array}$ & $\begin{array}{c}5.71 \\
(p<0.01)\end{array}$ & $\begin{array}{c}5.31 \\
(p<0.01)\end{array}$ \\
\hline$p=0.25$ & 60 & 60 & $\begin{array}{c}7.06 \\
(0.29)\end{array}$ & $\begin{array}{l}3.65 \\
(0.24)\end{array}$ & $\begin{array}{c}3.41 \\
(0.38)\end{array}$ & $\begin{array}{c}9.03 \\
(p<0.01)\end{array}$ & $\begin{array}{c}7.22 \\
(p<0.01)\end{array}$ \\
\hline$p=0.5$ & 55 & 60 & $\begin{array}{c}6.40 \\
(0.37)\end{array}$ & $\begin{array}{c}3.73 \\
(0.25)\end{array}$ & $\begin{array}{l}2.67 \\
(0.44)\end{array}$ & $\begin{array}{c}6.07 \\
(p<0.01)\end{array}$ & $\begin{array}{c}5.32 \\
(p<0.01)\end{array}$ \\
\hline$p=0.75$ & 54 & 56 & $\begin{array}{l}7.07 \\
(0.33)\end{array}$ & $\begin{array}{l}4.08 \\
(0.24)\end{array}$ & $\begin{array}{c}2.99 \\
(0.41)\end{array}$ & $\begin{array}{c}7.27 \\
(p<0.01)\end{array}$ & $\begin{array}{c}6.02 \\
(p<0.01)\end{array}$ \\
\hline $\begin{array}{l}\text { Difference: } \\
\quad(p=0.75)-(p=0)\end{array}$ & & & $\begin{array}{c}0.12 \\
(0.43)\end{array}$ & $\begin{array}{l}-0.37 \\
(0.43)\end{array}$ & $\begin{array}{c}0.49 \\
(0.61)\end{array}$ & $\begin{array}{c}0.80 \\
(p=0.42)\end{array}$ & \\
\hline $\begin{array}{l}\text { Panel B. Experiment } 2 \\
p=0.25\end{array}$ & 40 & 35 & $\begin{array}{c}5.99 \\
(0.32)\end{array}$ & $\begin{array}{c}3.24 \\
(0.33)\end{array}$ & $\begin{array}{c}2.74 \\
(0.47)\end{array}$ & $\begin{array}{c}5.89 \\
(p<0.01)\end{array}$ & $\begin{array}{c}4.89 \\
(p<0.01)\end{array}$ \\
\hline$p=0.5$ & 40 & 39 & $\begin{array}{c}6.03 \\
(0.33)\end{array}$ & $\begin{array}{l}3.97 \\
(0.34)\end{array}$ & $\begin{array}{l}2.05 \\
(0.47)\end{array}$ & $\begin{array}{c}4.34 \\
(p<0.01)\end{array}$ & $\begin{array}{c}4.25 \\
(p<0.01)\end{array}$ \\
\hline$p=0.75$ & 38 & 36 & $\begin{array}{c}5.41 \\
(0.32)\end{array}$ & $\begin{array}{c}4.64 \\
(0.34)\end{array}$ & $\begin{array}{c}0.77 \\
(0.47)\end{array}$ & $\begin{array}{c}1.64 \\
(p=0.10)\end{array}$ & $\begin{array}{c}1.58 \\
(p=0.11)\end{array}$ \\
\hline $\begin{array}{l}\text { Difference: } \\
\quad(p=0.75)-(p=0.25)\end{array}$ & & & $\begin{array}{l}-0.58 \\
(0.46)\end{array}$ & $\begin{array}{c}1.40 \\
(0.48)\end{array}$ & $\begin{array}{l}-1.98 \\
(0.66)\end{array}$ & $\begin{array}{c}2.99 \\
(p<0.01)\end{array}$ & \\
\hline $\begin{array}{l}\text { Panel C. Experiment } 3 \\
p=0.25\end{array}$ & 29 & 27 & $\begin{array}{c}5.21 \\
(0.49)\end{array}$ & $\begin{array}{c}2.98 \\
(0.40)\end{array}$ & $\begin{array}{c}2.23 \\
(0.64)\end{array}$ & $\begin{array}{c}3.49 \\
(p<0.01)\end{array}$ & $\begin{array}{c}3.58 \\
(p<0.01)\end{array}$ \\
\hline$p=0.5$ & 40 & 40 & $\begin{array}{c}4.88 \\
(0.39)\end{array}$ & $\begin{array}{c}3.19 \\
(0.37)\end{array}$ & $\begin{array}{l}1.69 \\
(0.54)\end{array}$ & $\begin{array}{c}3.15 \\
(p<0.01)\end{array}$ & $\begin{array}{c}3.22 \\
(p<0.01)\end{array}$ \\
\hline$p=0.75$ & 28 & 29 & $\begin{array}{c}5.66 \\
(0.37)\end{array}$ & $\begin{array}{c}2.91 \\
(0.27)\end{array}$ & $\begin{array}{l}2.75 \\
(0.46)\end{array}$ & $\begin{array}{c}5.98 \\
(p<0.01)\end{array}$ & $\begin{array}{c}4.95 \\
(p<0.01)\end{array}$ \\
\hline$p=0.99$ & 21 & 23 & $\begin{array}{c}6.21 \\
(0.53)\end{array}$ & $\begin{array}{c}4.09 \\
(0.35)\end{array}$ & $\begin{array}{l}2.13 \\
(0.63)\end{array}$ & $\begin{array}{c}3.38 \\
(p<0.01)\end{array}$ & $\begin{array}{c}3.35 \\
(p<0.01)\end{array}$ \\
\hline $\begin{array}{l}\text { Difference: } \\
\qquad(p=0.99)-(p=0.25)\end{array}$ & & & $\begin{array}{c}1.01 \\
(0.65)\end{array}$ & $\begin{array}{c}1.11 \\
(0.64)\end{array}$ & $\begin{array}{l}-0.10 \\
(0.91)\end{array}$ & $\begin{array}{c}0.11 \\
(p=0.91)\end{array}$ & \\
\hline
\end{tabular}

Notes: Standard errors are in parentheses. WTA $=$ Seller's Willingness To Accept. $W T P=$ Buyer's Willingness To Pay. $t$-test for equality in means. MW-test for equality in distributions.

magnitude of the endowment effect across conditions, $t=0.11,(p=0.91)$. Given both buyers and sellers exhibit sharp increases in valuations as $p$ increases from 0.75 to 0.99 , the positive result for buyers seems likely to be driven by the common design feature rather than the forces of the KR model in this setting. Ignoring the $p=0.99$ condition, WTA and WTP are effectively flat with respect to changes in $p$.

\section{Conclusion}

An important advance in the study of reference-dependent behavior is the discipline provided by structured mechanisms for determining the reference point. Köszegi and Rabin (2006) provide such discipline by positing a reference point grounded in rational expectations. The key construct in this development is that individuals rationally forecast their sensations of gains and losses and 
build consistent plans accordingly. This behavior is summarized in the rational expectations equilibrium concept, personal equilibrium, which states an individual can only expect to consume something that he will consume, given he expects it.

We examine the predictions of Kôszegi and Rabin (2006) personal equilibrium in the context of three exchange experiments with probabilistic forced exchange. Sellers endowed with university mugs and buyers endowed with money have their valuations elicited as in standard exchange experiments, but with one critical innovation. With probability $p$, exchange is forced at an arbitrary price regardless of stated preferences. This changes the expectations of the sellers (and buyers), they can no longer expect to hold on to their mug (not buy a mug), and consequently, their reference point should adjust. With the mug probabilistically gone (bought), selling (buying) feels less like a loss. This constrains personal-equilibrium behavior of not exchanging, making individuals more favorable to exchange the higher the probability that exchange will be forced. This insight leads to an asymmetric comparative static prediction. Buyers' Willingness to Pay (WTP) should increase with $p$, while sellers' Willingness to Accept (WTA) should decrease with $p$. This mechanism has the potential to eliminate and even reverse the commonly found endowment effect exchange asymmetry (Knetsch and Sinden 1984; Knetsch 1989; Kahneman, Knetsch, and Thaler 1990).

Our results highlight the sensitivity of KR predictions to plausibly minor changes in experimental design. Across our three experiments, six comparative statics are tested. Three of these tests are directionally consistent with the KR theory (buyers and sellers in experiment 2 , buyers in experiment 3 ), two with statistical significance (buyers in experiments 2 and 3). The asymmetric predictions for buyers and sellers are only observed simultaneously in experiment 2 . Even in the more tightly controlled experiments 2 and 3, the results are best described as mixed.

Though expectations-based reference dependence carries the promise of disciplining models of loss aversion, our results point to a clear challenge of grounding reference points in rational expectations. We are not alone in highlighting this sensitivity. In exchange behavior with probabilistic permission to trade, Ericson and Fuster (2011); Camerer et al. (2016), and Heffetz and List (2014), reach differing conclusions with quite similar designs. In experiments examining reference dependence and labor supply, Abeler et al. (2011) and Gneezy et al. (2017) also demonstrate the sensitivity of KR predictions to relatively small design differences. One potential conclusion is that though expectations-based reference points may help to understand economic behavior, they may be both hard to establish and fragile to minute changes in the economic environment.

Even when supportive of the directional predictions of the KR model, our results broadly reject the model's point predictions. Endowment effects exist in all of our experiments, at all probabilities of forced exchange. This suggests that other forces or points of reference beyond those of a strictly read KR model may be driving valuations. Our data would be particularly well accommodated by additionally considering the standard status quo reference point, which would predict an endowment effect at all probabilities. This echoes a number of recent contributions that organize behavior around plausible, non-expectations-based reference points (Allen et al. 2017; DellaVigna et al. 2017; Rees-Jones 2018). Of course, considering 
additional reference points leaves reference dependence without the discipline provided by the KR modeling advances. The value of KR lies in the researchers' ability to see the economic environment and immediately know the reference point, removing the powerful degree of freedom provided by an ad hoc formulation. Critical future steps will deliver understanding on when and why specific formulations of reference points are likely to be adopted by decision makers, perhaps giving the promise of regaining this discipline once again.

\section{Mathematical ApPendix}

\section{A. Personal Equilibrium with General $\mu$-function}

It is straightforward to show that keeping the mug is a PE for sellers also with a more generic function for the reference dependent utility that respects the property $\mu(Z)-\mu(-Z)<0$. To show this, consider the following argumentation for a mug seller:

$$
\begin{aligned}
U((M, 0) \mid(M, 0)) & =M+\mu(0), \\
U((0, x) \mid(M, 0)) & =x+\mu(-M)+\mu(x) .
\end{aligned}
$$

Keeping the mug is hence a PE if

$$
\begin{aligned}
U((M, 0) \mid(M, 0)) & \geq U((0, x) \mid(M, 0)), \\
M-\mu(-M) & \geq x+\mu(x),
\end{aligned}
$$

which, with $M=x$, implies $\mu(-x)+\mu(x)<0$, and hence $\bar{S}>M$, for any degree of loss aversion. Along the same lines can be shown that not buying is a PE for buyers for any degree of loss aversion.

\section{B. Personal Equilibrium with General $\mu$-function and Probabilistic Forced Exchange}

We can generalize the results from Section I using the same generic $\mu$-function as above. It is a PE for sellers to keep the mug as long as

$$
\begin{aligned}
& U(p \cdot(0, x)+(1-p) \cdot(M, 0) \mid p \cdot(0, x)+(1-p) \cdot(M, 0)) \\
& =p[x+(1-p)\{\mu(-M)+\mu(x)\}+p \mu(0)] \\
& \quad+(1-p)[M+(1-p) \mu(0)+p\{\mu(M)+\mu(-x)\}]
\end{aligned}
$$


and

$$
\begin{aligned}
& U(1 \cdot(0, x) \mid p \cdot(0, x)+(1-p) \cdot(M, 0)) \\
& \quad=x+(1-p)\{\mu(-M)+\mu(x)\}+p \mu(0) .
\end{aligned}
$$

Not selling is a PE as long as the first expression exceeds the second:

$$
\begin{gathered}
p[x+(1-p)\{\mu(-M)+\mu(x)\}]+(1-p)[M+p\{\mu(M)+\mu(-x)\}] \\
\geq x+(1-p)\{\mu(-M)+\mu(x)\}, \\
M+p\{\mu(M)+\mu(-x)\}-(1-p)\{\mu(-x)+\mu(x)\} \geq x .
\end{gathered}
$$

Assuming again $x=M$ :

$$
\begin{aligned}
p\{\mu(x)+\mu(-x)\}+(1-p)\{\mu(-x)+\mu(x)\} & \geq 0, \\
2 p(\mu(x)+\mu(-x)) & \geq \mu(x)+\mu(-x),
\end{aligned}
$$

and since $\mu(x)+\mu(-x)<0$, this leads us to the following three conditions for the predicted endowment effect:

$$
\begin{aligned}
& 2 p<1 \Leftrightarrow p<0.5, \bar{S}>M, \text { there is an endowment effect for } p<0.5 ; \\
& 2 p=1 \Leftrightarrow p=0.5, \bar{S}=M, \text { there is no endowment effect for } p=0.5 ; \\
& 2 p>1 \Leftrightarrow p>0.5, \bar{S}<M, \text { there is a reverse endowment effect for } p>0.5 .
\end{aligned}
$$

The argument is the same for buyers.

\section{Equilibrium Refinement}

The Choice-Acclimating Personal Equilibrium (CPE) compares all strategies and selects the one with the highest ex ante utility. Consider a potential mug seller first. A mug seller has two potential strategies: trying to hold onto her mug or choosing to sell it. CPE compares the values $U(p \cdot(0, x)+(1-p) \cdot(M, 0) \mid p \cdot(0, x)+(1-p) \cdot(M, 0))$ and $U((0, x) \mid(0, x))$ without appeal to whether these strategies are PE. From our previous derivations we know that the former yields

$$
p x+(1-p) M+p(1-p) \eta(1-\lambda)(M+x) .
$$

The latter yields $x$, alone. 
In order to choose which strategy to select, we compare these ex ante utilities. Whether a seller sells or not depends on the inequality

$$
p x+(1-p) M+p(1-p) \eta(1-\lambda)(M+x) \gtreqless x .
$$

Separating the terms in $M$ and $x$ yields

$$
x \gtreqless M \frac{1+p \eta(1-\lambda)}{1-p \eta(1-\lambda)} .
$$

Notice that the fraction on the right-hand side of the previous equation is less than 1 if $\eta>0$ and $\lambda>1$. This reflects the intuition behind the CPE in this case: because of the forced-exchange mechanism, the seller is willing to switch to selling "at a discount," i.e., even when $x$ is less than $M$, with the precise discount determined in the equation. Switching to selling frees the seller from any possible gain/loss sensations that reduce his utility. Therefore, it is rational to switch to the selling equilibrium even at prices below the intrinsic value.

A parallel argument, with the reverse implication, applies to buyers. They are willing to switch to buying "at a markup," i.e., even when the price exceeds the intrinsic value of the mug because it frees them from the possible gain/loss sensation from resisting buying. Analogous calculations show that a buyer switches between buying and not-buying when

$$
x \gtreqless M \frac{1-p \eta(1-\lambda)}{1+p \eta(1-\lambda)} .
$$

These two equations are the lines separating CPE values (for each buyers and sellers, respectively) plotted in Figure 2, panel A in the main text.

The Preferred Personal Equilibrium (PPE) refinement follows an identical logic but requires $U(p \cdot(0, x)+(1-p) \cdot(M, 0) \mid p \cdot(0, x)+(1-p) \cdot(M, 0))$ and $U((0, x) \mid(0, x))$ to be PE. First consider sellers. Recall that

$$
\underline{S}(p)=M \frac{1+\eta}{1+\eta \lambda} .
$$

If $x<\frac{1+\eta}{1+\eta \lambda} M$, selling is no longer PE. Hence, a seller can support selling in PPE at $x$ if

$$
x>M \frac{1+p \eta(1-\lambda)}{1-p \eta(1-\lambda)} \geq M \frac{1+\eta}{1+\eta \lambda} .
$$

The PPE strategies follows the CPE strategies until $p$ increases such that

$$
M \frac{1+p \eta(1-\lambda)}{1-p \eta(1-\lambda)}<M \frac{1+\eta}{1+\eta \lambda}
$$


and then follows $\underline{S}(p)$.

Similarly, consider buyers. Recall that

$$
\bar{B}(p)=M \frac{1+\eta \lambda}{1+\eta} .
$$

If $x>\frac{1+\eta \lambda}{1+\eta} M$, buying is no longer PE. Hence, a buyer can support buying in PPE at $x$ if

$$
x<M \frac{1-p \eta(1-\lambda)}{1+p \eta(1-\lambda)} \leq M \frac{1+\eta \lambda}{1+\eta} .
$$

The PPE strategies follow the CPE strategies until $p$ increases such that

$$
M \frac{1-p \eta(1-\lambda)}{1+p \eta(1-\lambda)}>M \frac{1+\eta \lambda}{1+\eta}
$$

and then follows $\bar{B}(p)$. The PPE cutoffs are plotted as the kinked lines in Figure 2, panel $\mathrm{A}$ in the main text.

\section{PE in Price List Lottery}

Previous analyses looked at PE for a given price. We now extend these arguments to let valuations interact with the method of choice. If individuals form valuations on a price list from which the valid price is drawn using a uniform distribution, the price itself becomes a lottery that influences expectations of trade. For the sake of demonstration, we consider a simplified price list with just three possible prices and assume the existence of a $\Delta$ big enough such that at price $x-\Delta$ a buyer wants to buy the mug under Strategy $K$. Where $K=$ \{buy, not buy, not buy and the deviation strategy $K^{\prime}=\{$ buy, buy, not buy $\}$. This leads to the following buying pattern:

\begin{tabular}{lcc} 
& Not buy & Buy \\
\hline$x-\Delta$ & & $K, K^{\prime}$ \\
$x$ & $K$ & $K^{\prime}$ \\
$x+\Delta$ & $K, K^{\prime}$ &
\end{tabular}

Each row occurs with a probability of $1 / 3$.

We only compare behavior under $K$ and $K^{\prime}$ at price $x$. When agents are not forced to exchange, all remaining terms are identical and cancel out of the equilibrium analysis. Utility of not buying the mug at price $x$ given his reference point is $K$ is

$$
U(K \mid K)=\frac{1}{3}[-\eta \lambda M+\eta(x-\Delta)]+\frac{1}{3} p(-\eta \lambda M+\eta x)+\frac{1}{3} p(-\eta \lambda M+\eta(x+\Delta)) .
$$


Utility of buying the mug at price $x$ given his reference point is $K$ is

$$
U\left(K^{\prime} \mid K\right)=M-x-\frac{1}{3} \eta \lambda \Delta+\frac{1}{3}(1-p)(\eta M-\eta \lambda x)+\frac{1}{3}[(1-p)(\eta M-\eta \lambda x)+p \eta \Delta] .
$$

The lowest price at which the buyer can support not buying the mug in PE is

$$
\begin{gathered}
x\left(1+\frac{2}{3} p \eta+\frac{1}{3} \eta+\frac{2}{3}(1-p) \eta \lambda\right)=M\left(1+\frac{2}{3}(1-p) \eta+\frac{2}{3} p \eta \lambda+\frac{1}{3} \eta \lambda\right)+\Delta \eta \frac{1}{3}(\lambda-1), \\
\underline{B}(p)=M \frac{1+\frac{2}{3}(1-p) \eta+\frac{2}{3} p \eta \lambda+\frac{1}{3} \eta \lambda}{1+\frac{2}{3} p \eta+\frac{1}{3} \eta+\frac{2}{3}(1-p) \eta \lambda}-\Delta \frac{1}{3} \eta \frac{(\lambda-1)}{1+\frac{2}{3} p \eta+\frac{1}{3} \eta+\frac{2}{3}(1-p) \eta \lambda} .
\end{gathered}
$$

A similar argument for sellers yields the following as the highest price at which she can support not selling the mug in PE:

$$
\bar{S}(p)=M \frac{1+\frac{2}{3}(1-p) \eta \lambda+\frac{2}{3} p \eta+\frac{1}{3} \eta}{1+\frac{2}{3} p \eta \lambda+\frac{1}{3} \eta \lambda+\frac{2}{3}(1-p) \eta}+\Delta \frac{1}{3} \eta p \frac{(\lambda-1)}{1+\frac{2}{3} p \eta \lambda+\frac{1}{3} \eta \lambda+\frac{2}{3}(1-p) \eta} .
$$

The comparative statics remain unchanged. The forced exchange makes buyers and sellers more inclined toward exchange, the price list merely amplifies the effects. A reverse endowment effects is already predicted at $p=0.5$ with $\underline{B}(p)>\bar{S}(p) \forall \lambda>1$.

\section{REFERENCES}

Abeler, Johannes, Armin Falk, Lorenz Goette, and David Huffman. 2011. "Reference Points and Effort Provision." American Economic Review 101 (2): 470-92.

Allen, Eric J., Patricia M. Dechow, Devin G. Pope, and George Wu. 2017. "Reference-Dependent Preferences: Evidence from Marathon Runners." Management Science 63 (6): 1657-72.

Bell, David E. 1985. "Disappointment in Decision Making under Uncertainty." Operations Research 33 (1): 1-27.

Camerer, Colin F., Anna Dreber, Eskil Forsell, Teck-Hua Ho, Jürgen Huber, Magnus Johannesson, Michael Kirchler, et al. 2016. "Evaluating replicability of laboratory experiments in economics." Science 351 (6280): 1433-36.

Cerulli-Harms, Annette, Lorenz Goette, and Charles Sprenger. 2019. "Randomizing Endowments: An Experimental Study of Rational Expectations and Reference-Dependent Preferences: Dataset." American Economic Journal: Microeconomics. https://doi.org/10.1257/mic.20170271.

DellaVigna, Stefano, Attila Lindner, Balázs Reizer, and Johannes F. Schmieder. 2017. "Reference-Dependent Job Search: Evidence from Hungary." Quarterly Journal of Economics 132 (4): 1969-2018.

de Meza, David, and David C. Webb. 2007. "Incentive Design under Loss Aversion." Journal of the European Economic Association 5 (1): 66-92.

Ericson, Keith M. Marzilli, and Andreas Fuster. 2011. "Expectations as Endowments: Evidence on Reference-Dependent Preferences from Exchange and Valuation Experiments." Quarterly Journal of Economics 126 (4): 1879-1907.

Gneezy, Uri, Lorenz Goette, Charles Sprenger, and Florian Zimmermann. 2017. "The Limits of Expectations-Based Reference Dependence." Journal of the European Economic Association 15 (4): 861-76. 
Greiner, Ben. 2004. "An Online Recruitment System for Economic Experiments.” Forschung und Wissenschaftliches Rechnen GWDG-Bericht 63.

Gul, Faruk. 1991. "A Theory of Disappointment Aversion.” Econometrica 59 (3): 667-86.

Heffetz, Ori, and John A. List. 2014. "Is the Endowment Effect an Expectations Effect?" Journal of the European Economic Association 12 (5): 1396-1422.

Heidhues, Paul, and Botond Kốszegi. 2008. "Competition and Price Variation When Consumers Are Loss Averse.” American Economic Review 98 (4): 1245-68.

Heidhues, Paul, and Botond Kőszegi. 2014. "Regular prices and sales." Theoretical Economics 9 (1): 217-51.

Herweg, Fabian, Daniel Müller, and Philipp Weinschenk. 2010. "Binary Payment Schemes: Moral Hazard and Loss Aversion." American Economic Review 100 (5): 2451-77.

Kahneman, Daniel, Jack L. Knetsch, and Richard H. Thaler. 1990. "Experimental Tests of the Endowment Effect and the Coase Theorem." Journal of Political Economy 98 (6): 1325-48.

Knetsch, Jack L. 1989. "The Endowment Effect and Evidence of Nonreversible Indifference Curves." American Economic Review 79 (5): 1277-84.

Knetsch, Jack L., and J. A. Sinden. 1984. "Willingness to Pay and Compensation Demanded: Experimental Evidence of an Unexpected Disparity in Measures of Value." Quarterly Journal of Economics 99 (3): 507-21.

Kőszegi, Botond, and Matthew Rabin. 2006. "A Model of Reference-Dependent Preferences." Quarterly Journal of Economics 121 (4): 1133-65.

Kőszegi, Botond, and Matthew Rabin. 2007. "Reference-Dependent Risk Attitudes." American Economic Review 97 (4): 1047-73.

Kőszegi, Botond, and Matthew Rabin. 2009. "Reference-Dependent Consumption Plans." American Economic Review 99 (3): 909-36.

Loomes, Graham, and Robert Sugden. 1986. "Disappointment and Dynamic Consistency in Choice under Uncertainty." Review of Economic Studies 53 (2): 271-82.

Mazar, Nina, Botond Köszegi, and Dan Ariely. 2014. "True Context-dependent Preferences? The Causes of Market-dependent Valuations." Journal of Behavioral Decision Making 27 (3): 200-208.

Pagel, Michaela. 2016. "Expectations-Based Reference-Dependent Preferences and Asset Pricing." Journal of the European Economic Association 14 (2): 468-514.

Pagel, Michaela. 2017. "Expectations-Based Reference-Dependent Life-Cycle Consumption." Review of Economic Studies 84 (2): 885-934.

Plott, Charles R., and Kathryn Zeiler. 2005. "The Willingness to Pay-Willingness to Accept Gap, the 'Endowment Effect,' Subject Misconceptions, and Experimental Procedures for Eliciting Valuations." American Economic Review 95 (3): 530-45.

Plott, Charles R., and Kathryn Zeiler. 2007. "Exchange Asymmetries Incorrectly Interpreted as Evidence of Endowment Effect Theory and Prospect Theory?" American Economic Review 97 (4): 1449-66.

Rees-Jones, Alex. 2018. "Quantifying Loss-Averse Tax Manipulation.” Review of Economic Studies 85 (2): 1251-78.

Sprenger, Charles. 2015. "An Endowment Effect for Risk: Experimental Tests of Stochastic Reference Points." Journal of Political Economy 123 (6): 1456-99. 